\title{
David Lindsay et The Haunted Woman
}

\section{Bernard Sellin}

\section{OpenEdition}

1 Journals

\section{Édition électronique}

URL : http://journals.openedition.org/etudesecossaises/3417

DOI : 10.4000/etudesecossaises.3417

ISSN : 1969-6337

\section{Éditeur}

UGA Éditions/Université Grenoble Alpes

\section{Édition imprimée}

Date de publication : 1 mars 2001

Pagination : 75-82

ISBN : 978-2-84310-198-4

ISSN : 1240-1439

\section{Référence électronique}

Bernard Sellin, «David Lindsay et The Haunted Woman », Études écossaises [En ligne], 7 | 2001, mis en ligne le 29 mars 2018, consulté le 08 septembre 2020. URL : http://journals.openedition.org/ etudesecossaises/3417; DOI : https://doi.org/10.4000/etudesecossaises.3417 


\section{David Lindsay et The Haunted Woman}

Les amateurs de littérature fantastique, surtout s'ils sont Écossais, connaissent David Lindsay (1876-1945) - tout au moins son premier livre $A$ Voyage to Arcturus (1920) sur lequel repose de manière quasi exclusive la réputation de l'auteur.

On connaît moins le reste de l'œuvre, une demi-douzaine d'ouvrages qui ont en commun de se situer aux lisières du fantastique, de la science-fiction ou du merveilleux. Rares sont les études portant sur ces ouvrages, il est vrai, moins éblouissants qu'Arcturus. Néanmoins ils constituent dans leur ensemble une œuvre solidement ancrée dans le fantastique. Si l'on excepte l'ouvrage peu représentatif The Adventures of Monsieur de Mailly (1926), tous les autres révèlent au lecteur des modes de communication peu ordinaires à la découverte d'une réalité suprasensible qui représente bel et bien le fondement de l'œuvre. Une prose de plus en plus pesante, des intrigues souvent peu crédibles, une volonté didactique envahissante ont éloigné critiques et lecteurs pour ne retenir finalement que les éclairs d'Arcturus.

Un titre a pourtant été injustement délaissé, The Haunted Woman (1922), que Lindsay rédige dans la fièvre qui accompagne la publication d'Arcturus, même si ce dernier ne connaît pas à l'époque le succès escompté. Par la suite les déceptions littéraires viendront tempérer l'enthousiasme de l'auteur.

The Haunted Woman parut en 1922, soit deux années après Arcturus. Les deux romans ont cependant peu de points communs et le lecteur qui se tournerait vers The Haunted Woman avec le secret espoir de prolonger l'expérience de $A$ Voyage to Arcturus ne tarderait pas à avouer sa déception. Nul voyage dans l'espace ici, pas d'êtres extraordinaires, pas d'aventures haletantes. Tout au plus un décor qui, aux premières pages, peut rappeler l'introduction d'Arcturus: la bourgeoisie aisée et oisive

BERNARd SELlin Université de Bretagne occidentale. qui fréquente les hôtels de Brighton dans la sérénité retrouvée du lendemain de la Première Guerre mondiale. Mais tandis 
que dans le premier roman l'intrigue bifurque de manière assez inattendue vers les grands espaces sidéraux, dans The Haunted Woman elle demeure irrémédiablement collée à la Terre, voire au terre à terre, puisqu'elle a pour point de départ un achat immobilier, en l'occurrence un vieux manoir blotti dans quelque vallon du Sussex. L'héroïne, Isbel Loment, est en fait la nièce de l'acheteur, Mrs Moor. On pourrait être dans un roman de Henry James si le mode de narration ne le démentait pas aussitôt. Cette Isbel est-elle apparentée à une autre héroïne célèbre du même nom, Isabel Archers de Portrait of $a$ $L a d y{ }^{1}$ ? Cela se pourrait bien même si les indices sont rares: un même penchant pour le fantastique et un chassé-croisé entre Amérique et Europe que ne renierait pas James. Toujours estil que c'est bien un "portrait de femme» que brosse Lindsay dans ce livre avec les moyens qui sont les siens. Portrait qui n'a pas la complexité de l'héroïne de James mais qui pose d'emblée que la nature humaine est double, voire indéchiffrable.

Ainsi Lindsay inscrit-il son roman dès les premières pages dans la dialectique du double déjà reconnaissable dans Arcturus. The Haunted Woman s'ouvre sur un monde d'apparences (tenues de soirée raffinées, bienséances mondaines, galanterie de règle) que l'intrusion du fantastique va bientôt faire voler en éclats.

Déjà aux premières pages quelques signes semés çà et là laissent entrevoir les failles de ce monde un peu trop lisse, à l'image du visage de l'héroïne maquillé à l'excès. "The tranquillity of her expression was rarely broken by an emotion or a smile, but whenever this did happen it was like a mask lifting.» (8) peut-on lire ${ }^{2}$. Et malgré cette tranquillité d'apparence, il y a chez elle une nervosité qui l'empêche de se tenir immobile.

Avouons le cependant, nous sommes loin d'éprouver à ce stade ce que Louis Vax appelle la «séduction de l'étrange» ${ }^{3}$.

1. Est-ce un autre hasard? HenryJames est mort quelques années auparavant, en 1916, non loin du lieu où Lindsay situe son intrigue, à Rye dans le Sussex.

2. L'édition utilisée est celle des éditions Gollancz, 1964. Tous les renvois de pages concernent cette édition.

3. Louis Vax, La Séduction de l'étrange (1965), PUF, Paris, $2^{\mathrm{e}}$ édition, 1987.
Toute l'habileté de Lindsay réside dans cette imperceptible transition à la rencontre du fantastique, non pas dans la brutale rupture de $A$ Voyage to Arcturus. Dans The Haunted Woman on entre dans le fantastique - et il s'agit bien de fantastique et non pas de science-fiction - par étapes successives et graduées.

L'ouvrage s'intitule The Haunted Woman mais il pourrait très bien s'appeler The Haunted House puisqu'il s'agit bien d'une histoire de maison hantée, dans une tradition écossaise, pourraiton dire si la maison ne constituait pas le lieu fantastique par excellence d'Edgar Poe à W.H. Hodgson entre autres. Maison 
hantée et bien évidemment maison ancienne tant il est vrai que le fantastique de Lindsay ne s'accommode guère de modernité, dans ce roman comme dans d'autres (Devil's Tor, The Violet Apple). À l'insignifiance de l'hôtel, lieu d'errance, s'oppose cette vieille demeure qui est l'objet de la transaction et dont l'origine remonterait au $\mathrm{VI}^{\mathrm{e}}$ siècle. Lieu fantastique par excellence, la maison donne accès à un univers à la fois enchanté et inquiétant dans lequel les normes de la vie de tous les jours n'ont plus cours. C'est l'une des fonctions du surnaturel dans ce livre : à la platitude, aux conventions et au matérialisme de la vie sociale ordinaire s'opposent des relations en apparence plus vraies, plus profondes et passionnées. En apparence seulement et nous y reviendrons.

Pour ce faire Lindsay a recours à l'escalier magique que d'autres ont utilisé avant lui, un escalier qui n'apparaît qu'à certaines personnes seulement et dont l'at trait est irrésistible. Revenu à la vie normale, l'individu n'a aucun souvenir de cette aventure extraordinaire.

Rien de sordide dans cette histoire qui n'évoque guère les contes répugnants de Lovecraft ou de Machen. La ville a renoncé à être un espace fantastique pour incarner au contraire les valeurs bourgeoises bienséantes. La nature ne participe pas vraiment au bouleversement même si le manoir en question est isolé, perdu au fond de la campagne. Ici la nature reste le lieu de l'insouciance propice au pique-nique. G'est donc dans la maison que se concentre le caractère fantastique: une demeure cossue, en bon état, récemment rénovée, qui ne suscite nulle inquiétude, seulement un sentiment de respect à l'égard d'une habitation chargée d'histoire.

Il ne s'agit donc nullement d'un univers a priori bizarre ou inquiétant, mais plutôt de l'intrusion inattendue du surnaturel dans la réalité quotidienne. Il est vrai que seuls quelques êtres d'exception ont la faculté de communiquer avec l'au-delà, suggère Lindsay. Tous les personnages du livre n'ont pas cette chance, si l'on peut dire, et l'auteur n'hésite pas à ranger son héroïne au nombre des Celtes visionnaires familiers des esprits par opposition aux Saxons jugés matérialistes et terre-à-terre. De quels Geltes s'agit-il? Nous ne le saurons jamais, mais il est

4. Le nom de l'héroïne interpelle quelque peu: Isbel Loment. Faut-il l'associer à des patronymes écossais tels que Lomond ou Lamont? permis de penser aux Gaëls d'Ecosse, évoqués dans d'autres ouvrages de Lindsay ${ }^{4}$. C'est d'ailleurs une des rares allusions à l'Écosse dans cet ouvrage situé dans le sud de l'Angleterre, a priori éloigné de toute contrée sous influence surnaturelle. 
L'expérience surnaturelle est décrite en plusieurs étapes correspondant à l'investigation du lieu magique. Trois portes closes recèlent des mystères, offrent des tentations auxquelles l'héroïne ne résiste pas, ce qui constitue les trois moments forts du livre. Le premier fait fonction d'introduction non seulement au monde magique mais encore sert de révélateur d'une personnalité double: derrière le moi social conventionnel se cache une nature profonde passionnée. La deuxième porte mène à une expérience qui malgré l'extrême chasteté de la description est de nature amoureuse. Que se passe-t-il derrière cette porte entre la jeune femme de 25 ans et le quinquagénaire propriétaire du lieu? "It was like awakening by degrees to the awful consciousness of having committed a crime during a period of temporary insanity" se dit la jeune femme avant de se reprendre: "Only it was not insanity [...] It sprang from the genuine and unfeigned emotion of both their hearts.» (119) Ainsi selon cette interprétation, le récit se lit comme une initiation (sexuelle?) d'autant plus impardonnable qu'Isbel est sur le point d'épouser un jeune homme de son âge. Il n'y a pas que le fantastique qui fait scandale. Isbel le rappelle assez souvent. Disons plutôt qu'à la rupture engendrée par l'intrusion du fantastique correspond une remise en question tout aussi radicale des normes sociales traditionnelles. Le fantastique, chez Lindsay, comme chez tant d'autres, est ce qui interpelle et fait problème. Il participe de la contestation des valeurs, notamment des valeurs héritées de la société bourgeoise traditionnelle. La rencontre dans la chambre «hantée» fait vaciller un projet de mariage et sème le trouble dans les esprits ${ }^{5}$.

Le plus étonnant reste encore à venir, derrière la troisième et dernière porte, celle qui dès le début semblait la plus menaçante. C'est le troisième mouvement de l'œuvre et celui qui mène à la conclusion. On s'éloigne alors d'un surnaturel somme toute assez banal pour plonger plus radicalement dans

5. Notons au passage que ce coup de foudre entre un quinquagénaire et une jeune femme de 25 ans correspond sensiblement au cas personnel de l'auteur dont le mariage tardif surprit son entourage. Voir Bernard Sellin, The Life and Works of David Lindsay, Cambridge University Press, Cambridge, 1981, p. 18-21. le merveilleux d'un jardin extraordinaire saturé de lumière et de musique, hanté par la présence d'un étrange musicien qui, semble-t-il, renvoie le lecteur à la philosophie pessimiste de $A$ Voyage to Arcturus. Désormais monde des esprits et réalité tangible s'interpénètrent sans qu'il soit toujours possible de les séparer. Le récit trouve son épilogue dans l'irruption du tragique et dans ce qui ressemble à l'effondrement des illusions. La fin du livre se prête à plusieurs interprétations tant la présentation demeure obscure. Il apparaît cependant que l'amour- 
passion entre Isbel et Henry Judge perd son aura romantique lorsqu'il devient probable que cet amour était en quelque sorte le résultat d'une manipulation, en l'occurrence celle du musicien, sorte de démon pervers s'adonnant au cruel passe-temps d'égarer les amants.

La fin du livre inscrit le récit dans la même dualité que celle déjà présente dans $A$ Voyage to Arcturus: le réel et le surnaturel se côtoient de façon déroutante. Automne et printemps se livrent à un chassé-croisé. Il y a deux femmes en Isbel Loment de même qu'il y a deux faces chez le musicien, l'une d'entre elles généralement occultée, car elle signifie la mort et la désillusion.

L'extrait suivant suggère encore l'utilisation que fait Lindsay de l'opposition des contraires, dans un passage qui ne peut manquer de rappeler certains chapitres d'Arcturus:

Well, then - when I was listening to that weird sound in that passage, it suddenly seemed to strike a very deep string in my heart, which had never been struck before. It was a kind of passion... It was passion. But there was something else in it besides joy - my heart felt sick and tormented, and there was a horrible sinking sensation of despair. But the delight was there all the time, and was the strongest... It only lasted a very short time, but I don't think I could forget it... (43)

Sous des dehors plus raffinés qu'Arcturus, The Haunted Woman explore des thèmes somme toute assez proches, notamment la dualité de l'existence. Le livre parvient remarquablement à mettre en évidence les deux plans: d'une part une vie conventionnelle, bourgeoise, superficielle, régie par des considérations matérielles telles que argent, demeure, mariage, et d'autre part la possibilité d'accéder à un autre mode d'existence décrit en termes magiques et marqué par des relations spirituelles profondes et passionnées. C'est ce qu'évoque l'air de musique qui parcourt le roman.

A tune was being played, so there was no doubt about the nature of the noise. It was a simple, early-English rustic air - sweet, passionate and haunting. The sonorous and melancholy character of the instrument added a wild, long-drawn-out charm to it which was altogether beyond the range of the understanding and seemed to belong to other days, when feelings were more poignant and delicate, less showy, splendid, and odourless... (127)

Lindsay exploite subtilement les transitions d'un mode d'existence à l'autre, déroutant ainsi les amants - et parfois ses 
lecteurs. Cependant, il ne fait guère de doute que le propos est de présenter à travers une fiction une philosophie de l'existence qui s'inspire de courants acceptant la validité d'une autre réalité supranaturelle. Se trouvent associés pêle-mêle les esprits, les rêves, les fantômes, la mort. Ainsi certains êtres auraient des facultés particulières leur permettant de communiquer avec ce monde des esprits. Dans The Haunted Woman, un personnage étranger au monde des amants parvient à s'introduire jusqu'à la chambre hantée uniquement grâce à ses dons de médium. Il s'agit de Mrs Richborough.

Le lecteur de ce début du $\mathrm{XXI}^{\mathrm{e}}$ siècle peut à juste titre contester de telles notions. Ceci dit, c'est bien l'utilisation littéraire qui nous intéresse et non pas la validité d'une quelconque communication surnaturelle. Pour Lindsay, en revanche, il ne fait guère de doute que ce monde suprasensible existe bel et bien comme il le confie à son ami E.H.Visiak: "We are surrounded by a terribly queer unseen universe ${ }^{6}$. D'autres remarques consignées dans ses carnets de notes le confirment.

Lindsay a désigné sous le nom de Sublime l'état d'existence originel selon lui, représentant un modèle idéal avant la dégradation que l'homme connaît aujourd'hui. Dans certaines circonstances, il nous arrive d'éprouver, selon Lindsay, une profonde nostalgie pour ce monde perdu. Vraisemblablement la chambre hantée du livre ne représente rien d'autre que ce monde idéal et l'on notera au passage quelques éléments fondamentaux qui le composent: la dimension surnaturelle, la profondeur passionnée des sentiments, l'intense spiritualité, l'union des principes de douleur et de plaisir, la musicalité.

À ce propos on ne peut manquer de remarquer l'omniprésence de la musique dans le roman. L'introduction du manoir hanté est rythmée par l'ouverture de la $7^{\mathrm{e}}$ Symphonie de Beethoven (36) comme pour installer le décor et suggérer par làmême que le récit aura pour pendant la musique, c'est à dire une forme d'expression qui mieux que toute autre suggère la beauté, la grandeur et la passion du Sublime. Il est clair que la musique de Beethoven d'entrée de jeu a pour fonction d'arra-

6. Lettre en date du 9 novembre 1921 in Adam International Review, vol XXXV, n 346-48, p. 43. 7. The Violet Apple and The Witch, Chicago Review Press, 1976, p. 281. cher le lecteur à la vie conventionnelle de tous les jours pour l'introduire dans l'autre monde sublime. Pour s'en persuader il suffit de se tourner vers un roman posthume, The Witch dans lequel on peut lire: «People were doomed to stay enchanted in the dream of life until aroused by the right music ${ }^{7} »$. 
Et encore dans les carnets de l'écrivain, cette fois-ci à propos de la $7^{\mathrm{e}}$ Symphonie: «Its true name is the Supernatural Symphony. Its character is peculiar, weird and mysterious: a long dream. One can hear it for ever and yet not understand it ${ }^{8}$.

Bientôt à Beethoven se substitue une musique plus discrète mais non moins captivante, interprétée sur un instrument ancien proche du violoncelle. Telle est la musique mystérieuse qui semble pousser Isbel et Henry Judge l'un vers l'autre.

The tone of the instrument, notwithstanding its small size, was midway in depth between that of a violoncello and that of a contra-bass, and the low, slow scrape of its strings had a peculiarly disturbing effect upon her feelings. The theme had a strange, archaic flavour, as though it had come down through centuries, yet it was so appropiate that Isbel could almost fancy it to be the voice of the landscape. It was hauntingly beautiful, and full of queer surprises; each long, sonorous note contained a world of music in itself, but it was powerful, yet delicate and passionate thought slowly being developed as the air proceeded which stirred her so exceedingly. (132)

Que représente ce musicien? Impossible d'éviter la question même si la réponse est délicate. Doit-on y voir le représentant du monde des esprits? L'explication est peu convaincante et incompatible avec la mort de Mrs Richborough, elle qui se targue de fréquenter les esprits sans crainte. S'agit-il de l'incarnation du Sublime, dans ce qu'il a d'insoutenable aux humains? C'est peu probable. Plus vraisemblablement, il s'agit d'un nouveau visage du Malin, familier des lecteurs d'Arcturus, le célèbre Crystalman ici déguisé en musicien. Ce musicien est toujours présenté de dos dans le livre et plusieurs indices laissent penser qu'il est à l'origine de la mystification dont les héros sont victimes. Les deux personnages qui ont l'audace de l'approcher de près et de découvrir son visage meurent peu après.

Comme l'ouvrage qui le précède, The Haunted Woman ne manque pas d'ambiguïté. Le lecteur peut à juste titre s'interroger sur l'interprétation des épisodes du livre mais là réside en grande partie l'intérêt. S'il est vrai, comme l'affirme Todorov ${ }^{9}$,

8. Philosophical notes, note 261. National Library of Scotland.

9. Tzvetan Todorov, Introduction à la littérature fantastique, Paris, Le Seuil, 1970, p. 36 . que l'essence du fantastique réside dans l'hésitation du lecteur face à ce qui lui est présenté comme étant la réalité, alors The Haunted Woman place le lecteur devant une double hésitation: hésitation à la fois face aux événements et à leur interprétation. Certains pourront le regretter, estimant que l'ouvrage 
manque de clarté, notamment dans sa conclusion. Pour notre part nous estimons que cette liberté d'interprétation constitue au contraire un atout, indissociable de la notion même de littérature. 